\title{
CLASSES OF OPERATORS ON VECTOR VALUED INTEGRATION SPACES
}

\author{
R. J. FLEMING and J. E. JAMISON \\ (Received 30 April; revised 20 October 1976) \\ Communicated by E. Strzelecki
}

\begin{abstract}
Let $L^{p}(\Omega, K)$ denote the Banach space of weakly measurable functions $F$ defined on a finite measure space and taking values in a separable Hilbert space $K$ for which $\|F\|_{p}=\left(\int|F(\omega)|^{p}\right)^{1 / p}<+\infty$. The bounded Hermitian operators on $L^{p}(\Omega, K)$ (in the sense of Lumer) are shown to be of the form$$
A F(\omega)=B(\omega) F(\omega),
$$

where $B(\omega)$ is a uniformly bounded Hermitian operator valued function on $K$. This extends the result known for classical $L^{p}$ spaces. Further, this characterization is utilized to obtain a new proof of Cambern's theorem describing the surjective isometries of $L^{p}(\Omega, K)$. In addition, it is shown that every adjoint abelian operator on $L^{p}(\Omega, K)$ is scalar.
\end{abstract}

Subject classification (Amer. Math. Soc. (MOS), 1970): primary 46 E 40, 46 E 30, 47 B 99

\section{Introduction}

The concept of a hermitian operator on a Banach space is due to G. Lumer (1961). Lumer (1963) characterized all bounded hermitian operators on a non-atomic reflexive Orlicz space as multiplications by real $L^{\infty}$ functions and used this characterization to determine the surjective isometries. The atomic case was treated by Tam (1969). Similar characterizations have been undertaken by the present authors for certain direct sums of Hilbert spaces (Fleming and Jamison, 1974a, b) and for $l^{p}$ sums of Banach spaces by Berkson and Sourour (1974). It is of interest then to consider the problem of finding the bounded hermitian operators on $L^{p}(\mu)$ spaces of vector valued functions when the measure is not necessarily purely atomic.

In the present paper we characterize the bounded hermitian operators on the spaces $L^{p}(\Omega, k)$ of weakly measurable functions with values in a separable Hilbert space. From these results we obtain a characterization of the surjective isometries which has been obtained also by Cambern (1974) with different methods. The

\section{(C) Copyright Australian Mathematical Society 1977}

Copyright. Apart from any fair dealing for scholarly purposes as permitted under the Copyright Act, no part of this JOURNAL may be reproduced by any process without written permission from the Treasurer of the Australian Mathematical Society. 
results on hermitian operators are also used to show that the adjoint abelian operators on $L^{p}(\Omega, k)$ are scalar. This is related to an open problem of Stampfli (1969).

Some comments are in order concerning the generality of the theorems proved in the paper. In the first place, standard adjustments in the arguments will allow the measure space $\Omega$ to be $\sigma$-finite. More delicate is the assumption that $K$ is a separable Hilbert space. Although the theorem is true if $K$ is assumed to be a separable smooth Banach space, we feel that the essential elements of the proof are best revealed and understood by assuming $K$ to be a Hilbert space. However, this last assumption seems to be essential for our proof of Theorem 4.14. More will be said on this subject in Section 4 .

\section{Hermitian operators and the spaces $L^{p}(\Omega, K)$}

For the definition of a semi-inner-product (abbreviated s.i.p.) we refer the reader to Lumer's paper (1961). A s.i.p. [,] on a complex Banach space $X$ is said to be compatible with the norm if $[F, F]=\|F\|^{2}$ for every $F \in X$. A bounded linear operator $T$ on a complex Banach space is said to be hermitian if there is a s.i.p. compatible with the norm, such that $[T F, F]$ is real for every $F \in X$. In the case that $X$ is a Hilbert space this definition yields the usual class of bounded hermitian operators.

Let $(\Omega, \Sigma, \mu)$ be a finite measure space and let $K$ be a separable Hilbert space with norm and inner product denoted by $|\cdot|_{2}$ and (,) respectively. A $K$ valued function $F$ on $\Omega$ is said to be weakly measurable (Hille and Phillips, 1957) if the complex valued function $(F(\cdot), z)$ is measurable for each $z \in K$. For $1 \leqslant p<\infty$, we denote by $L^{p}(\Omega, K)$ the Banach space of (equivalence classes of) weakly measurable $K$ valued functions on $\Omega$ for which $\|F\|_{p}<\infty$, where

$$
\begin{aligned}
& \|F\|_{p}=\left(\int|F(\omega)|_{2}^{p} d \mu\right)^{1 / p}, \quad p<\infty \\
& \|F\|_{\infty}=\operatorname{ess} \sup |F(\omega)|_{2} \text { for } p=\infty .
\end{aligned}
$$

It is important to note that the vector simple functions of the form

$$
\psi=\sum_{i=1}^{n} \chi_{E_{i}} x_{i}
$$

where the $E_{i}$ are disjoint measurable sets and the $x_{i}$ are distinct elements of $K$, are dense in $L^{p}(\Omega, K)$. For further facts concerning $L^{p}(\Omega, K)$ we refer the reader to Dunford and Schwartz (1958), Hille and Phillips (1957) and Lang (1969).

\section{Hermitian operators on $L^{p}(\Omega, K)$}

In what follows, the support of a $K$ valued function $F$ on $\Omega$ is the set $\{\omega \mid F(\omega) \neq 0\}$ where 0 is the zero vector in $K$, and if $z \in K, z$ is the function which is equal to $z$ for all $\omega \in \Omega$. We use script letters to denote operators on $L^{p}(\Omega, K)$. 
Since the dual space of $L^{p}(\Omega, K)$ for $1 \leqslant p<\infty$ is $L^{q}(\Omega, K)$ where $1 / p+1 / q=1$ (Lang, 1969), it is easy to show that a s.i.p. compatible with the norm on $L^{p}(\Omega, K)$, $1 \leqslant p<\infty$, is given by

$$
[F, G]=\int(F(\omega), G(\omega))\left(\frac{|G(\omega)|_{2}}{\|G\|_{p}}\right)^{p-2} d \mu .
$$

Before we state the main theorem on hermitian operators on $L^{p}(\Omega, K)$ we offer the following example of a bounded hermitian operator on $L^{p}(\Omega, K)$.

EXAMPLE: Let $H(\omega)$ be a $\mathscr{B}(K)$ valued function on $\Omega$ such that

(3.2) $H(\omega)=H(\omega)^{*}$ almost everywhere,

(3.3) $H(\omega) z$ is weakly measurable for each $z \in K$, and

(3.4) $\|H(\omega)\| \leqslant M<\infty$ almost everywhere, where $\|\cdot\|$ denotes the uniform norm on $\mathscr{B}(K)$. If we define $\left(\mathscr{M}_{H} F\right)(\omega)=H(\omega) F(\omega)$ then in view of (3.3) and (3.4) it is clear that $\mathscr{M}_{H}$ is a bounded linear operator on $L^{p}(\Omega, K)$. Furthermore, since

$$
\left[\mathscr{M}_{H} F, F\right]=\int(H(\omega) F(\omega), F(\omega))\left(\frac{|F(\omega)|_{2}}{\|F\|_{p}}\right)^{p-2} d \mu
$$

it follows from (3.2) that $\mathscr{M}_{H}$ is hermitian.

In this section we will show that every bounded hermitian operator on $L^{p}(\Omega, K)$ is of the form $\mathscr{H}_{H(.)}$ for some $\mathscr{B}(K)$ valued function $H(\cdot)$ satisfying the conditions of the example. To do this we first need to establish some preliminary results. Henceforth, $1 \leqslant p<\infty$ and $p \neq 2$.

(3.6) Lemma. Let $F_{1}, F_{2} \in L^{p}(\Omega, K)$ with disjoint supports $E_{1}$ and $E_{2}$. If $\mathscr{A}$ is a bounded hermitian operator on $L^{p}(\Omega, K)$ then

$$
\int_{E_{1}}\left(\left(\mathscr{A} F_{1}\right)(\omega), F_{2}(\omega)\right)\left|F_{2}(\omega)\right|_{2}^{p-2} d \mu=\overline{\int_{E_{1}}\left(\left(\mathscr{A} F_{2}\right)(\omega), F_{1}(\omega)\right)\left|F_{1}(\omega)\right|_{2}^{p-2} d \mu .}
$$

The proof of this lemma follows immediately by writing out the requirement that $\left[\mathscr{A}\left(F_{1}+e^{i \varphi} F_{2}\right), F_{1}+e^{i \varphi} F_{2}\right]$ is real for every choice of $\varphi \in \mathscr{R}$. We omit the details.

(3.8) Lemma. Let $E \in \Sigma$ with $\mu(E)>0$ and $z \in K$. If $\mathscr{A}$ is a bounded hermitian operator on $L^{p}(\Omega, K), 1 \leqslant p<\infty, p \neq 2$, then

$$
\mathscr{A}\left(\chi_{E} z\right)=\chi_{E} \mathscr{A}(z)
$$

Proof. It is enough to prove this for vector $z$ with $|z|_{2}=1$. Thus we let $z \in K$ with norm 1 and $E$ be a set in $\Sigma$ with positive measure. Let $E_{1}$ be any subset of $\Omega \backslash E$ with positive measure. Let $F_{1}=\chi_{E} z$ and $F_{2}=\alpha F$ where $\alpha$ is a non-zero real number and $F \in L^{p}(\Omega, K)$ with support in $E_{1}$ and $|F(\omega)|_{2}=1$ almost everywhere 
on $E_{1}$. Then from (3.7) we have

$$
\begin{aligned}
\int_{E_{1}}\left(\mathscr{A}\left(\chi_{E} z\right)(\omega), \alpha\right. & F(\omega))|\alpha F(\omega)|_{2}^{p-2} d \mu \\
= & \int_{E}\left(\alpha(\mathscr{A} F)(\omega), \chi_{E}(\omega) z\right) \mid \chi_{E}(\omega) z_{2}^{p-2} d \mu .
\end{aligned}
$$

For $\alpha=1$ then we have

$$
\int_{E_{1}}\left(\mathscr{A}\left(\chi_{E} z\right)(\omega), F(\omega)\right) d \mu=\overline{\int_{E}\left(\mathscr{A}(F)(\omega), \chi_{E}(\omega) z\right) d \mu .}
$$

Combining (3.10) and (3.11) we obtain

$$
\left(1-|\alpha|^{p-2}\right) \int_{E_{1}}\left(\mathscr{A}\left(\chi_{E} z\right)(\omega), F(\omega)\right) d \mu=0 .
$$

Since $p \neq 2$ we see from (3.12) that

$$
\int_{E_{1}}\left(\mathscr{A}\left(\chi_{E} z\right)(\omega), F(\omega)\right) d \mu=0 .
$$

If we now suppose that $E_{1}=\operatorname{supp}\left(\mathscr{A}\left(\chi_{E} z\right)\right) \cap(\Omega \backslash E)$ has positive measure, then from (3.13) we are forced to conclude that

$$
\int_{E_{1}}\left|\mathscr{A}\left(\chi_{E^{\prime}} z\right)(\omega)\right|_{2} d \mu=0
$$

Clearly this is impossible and we must conclude that the set $E_{1}$ has zero measure. Whence, the support of $\mathscr{A}\left(\chi_{E} z\right)$ is contained in $E$. The conclusion of the lemma is now easily seen.

With what we have established we will now show that a bounded hermitian operator on $L^{p}(\Omega, K), 1 \leqslant p<\infty, p \neq 2$, is induced by a $\mathscr{B}(K)$ valued multiplication.

In what follows we shall assume that $\mathscr{A}$ is a bounded hermitian operator on $L^{p}(\Omega, K), 1 \leqslant p<\infty, p \neq 2$. Thus

$$
\int|\mathscr{A}(F)(\omega)|_{2}^{p} d \mu \leqslant\|\mathscr{A}\|^{p}\|F\|_{p}^{p}
$$

for each $F \in L^{p}(\Omega, K)$. From (3.15) and (3.9) we see that for each $z \in K$ there exists a set $E_{z} \in \Sigma$ such that $\mu\left(E_{z}\right)=0$ and

$$
|\mathscr{A}(z)(\omega)|_{2} \leqslant\|\mathscr{A}\||z|_{2} \text { for every } \omega \in \Omega \backslash E_{z}
$$

Let $L$ be a countable dense subset of $K$. Then from (3.16) we see that there is a set $E_{0} \in \Sigma$ with $\mu\left(E_{0}\right)=0$ such that for any $z$ and $z^{\prime}$ in $L \cup\{0\}$

$$
\left|\mathscr{A}\left(z-z^{\prime}\right)(\omega)\right|_{2} \leqslant\|\mathscr{A}\|\left|z-z^{\prime}\right|_{2} \text { for } \omega \in \Omega \backslash E_{0} \text {. }
$$

If $\left(z_{n}\right)$ is a Cauchy sequence from $L \cup\{0\}$ then it is clear from (3.17) that $\left(\mathscr{A}\left(z_{n}\right)(\omega)\right)$ is a Cauchy sequence in $K$ for each $\omega \in \Omega \backslash E_{0}$. Since $L$ is dense any $z \in K$ is the limit of some sequence $\left(z_{n}\right)$ from $L$. Thus we define for each $\omega \in \Omega \backslash E_{0}$ and $z \in K$

$$
H(\omega) z=\lim \mathscr{A}\left(z_{n}\right)(\omega),
$$


where $\left(z_{n}\right)$ is a sequence from $L$ converging to $z$. It is easy to see that $H(\omega) z$ is well defined and we note that for each $z \in K, H(\cdot) z$ is a weakly measurable $K$ valued function. Furthermore, from (3.17) we have

$$
|H(\omega) z|_{2} \leqslant\|\mathscr{A}\||z|_{2} \text { for } \omega \in \Omega \backslash E_{0} .
$$

Whence $H(\cdot) z$ belongs to $L^{p}(\Omega, K)$ for each $z \in K$.

If $z \in K$ and $\left(z_{n}\right)$ is a sequence from $L$ which converges to $z$ then $\left|H(\omega) z-\mathscr{A}\left(z_{n}\right)\right|_{2}^{p}$ converges to zero for almost all $\omega$ and since it is dominated by an integrable function it follows by Lebesgue's dominated convergence theorem that

$$
\lim \left\|H(\cdot) z-\mathscr{A}\left(z_{n}\right)\right\|_{p}=0 .
$$

But since $\lim \left\|z-z_{n}\right\|_{p}=0$ we must have

$$
H(\cdot) z=\mathscr{A}(z)(\cdot) .
$$

In view of (3.21) we have established that for each $\omega \in \Omega \backslash E_{0}, H(\cdot)$ is a linear transformation on $K$. Moreover from (3.19) we have that $H(\omega)$ is bounded for each $\omega \in \Omega \backslash E_{0}$ and in fact

$$
\|H(\omega)\| \leqslant\|\mathscr{A}\| \text { for } \omega \in \Omega \backslash E_{0} .
$$

Therefore if we define $\mathscr{M}_{H(\cdot)}$ on $L^{p}(\Omega, K)$ by $\left(\mathscr{M}_{H(\cdot)} F\right)(\omega)=H(\omega) F(\omega)$ it will follow that $\mathscr{M}_{H(\cdot)}=\mathscr{A}$. To see this let $\psi=\sum_{i=1}^{n} \chi_{\mathbb{R}_{i}} z_{i}$, where the $z_{i}$ are distinct elements of $K$ and the $E_{i}$ are disjoint measurable sets. By the linearity of $\mathscr{A}$ and the definition of $\mathscr{M}_{H(.)}$ it is clear that

$$
\mathscr{M}_{H(.)} \psi=\mathscr{A} \psi \text {. }
$$

Since vector simple functions are dense in $L^{p}(\Omega, K)$ it follows that

$$
\mathscr{M}_{H(\cdot)} F=\mathscr{A} F \text { for each } F \in L^{p}(\Omega, K) \text {. }
$$

(3.25) THEOREM. Let $1 \leqslant p<\infty, p \neq 2$. Then $\mathscr{A}$ is a bounded hermitian operator on $L^{p}(\Omega, K)$ if and only if there is a $\mathscr{B}(K)$ valued function $H(\cdot)$ defined on $\Omega$ such that

(3.26) $H(\omega) z$ is weakly measurable for each $z \in K$.

(3.27) $\|H(\omega)\| \leqslant\|\mathscr{A}\|$ almost everywhere,

(3.28) $H(\omega)=H(\omega)^{*}$ almost everywhere,

(3.29) $\mathscr{A} F=\mathscr{M}_{H(\cdot)} F$ for $F \in L^{p}(\Omega, K)$.

Proof. The sufficiency is clear and in view of the preceding argument all that remains to be shown is (3.28). To this end let $z \in L$ with $|z|_{2}=1$. Then

$$
[\mathscr{A}(z), z]=\int(H(\omega) z, z) d \mu \text {. }
$$

Since $\mathscr{A}$ is hermitian, there is a set $E_{z} \in \Sigma$ with $\mu\left(E_{z}\right)=0$ such that $(H(\omega) z, z)$ is real for $\omega \in \Omega \backslash E_{z}$. But since $L$ is countable it follows that there exists a set $E_{0} \in \Sigma$ 
with $\mu\left(E_{0}\right)=0$ such that for each $z \in L$

$$
(H(\omega) z, z) \text { is real for } \omega \in \Omega \backslash E_{0} .
$$

Furthermore, since $L$ is dense and since the inner product is continuous on both variables (3.31) holds for each $z \in K$ and hence $H(\omega)=H(\omega)^{*}$ for $\omega \in \Omega \backslash E_{0}$. This completes the proof.

\section{The isometries of $L^{p}(\Omega, K)$}

In what follows we shall assume that $\mathscr{W}$ is a surjective isometry of $L^{p}(\Omega, K)$. If $T \in \mathscr{B}(K)$ we denote by $\mathscr{M}_{T}$ the operator defined by $\left(\mathscr{M}_{T} F\right)(\omega)=T[F(\omega)]$ for $F \in L^{p}(\Omega, K) . \mathscr{M}_{T}$ is a bounded linear operator and is hermitian on $L^{p}(\Omega, K)$ whenever $T=T^{*}$. We note that $\mathscr{H}_{T}=\mathscr{H}_{T_{1}}+i \mathscr{H}_{T_{2}}$ where $T_{1}=T_{1}^{*}, T_{2}=T_{2}^{*}$ and $T=T_{1}+i T_{2}$. Similarly if $E \in \Sigma$ we denote by $\mathscr{C}_{E}$ the operator on $L^{p}(\Omega, K)$ defined by

$$
\left(\mathscr{C}_{E} F\right)(\omega)=\chi_{E} F(\omega) .
$$

The operator $\mathscr{C}_{E}$ is a hermitian projection on $L^{p}(\Omega, K)$. It is clear that $\mathscr{W} \mathscr{C}_{E} \mathscr{W}^{-1}$ is a projection and by a result of Koehler and Rosenthal (1970) it is also hermitian. We can say more.

(4.2) Lemma. For each $E \in \Sigma$ there exists a set $\Phi(E) \in \Sigma$ such that

$$
\mathscr{W}_{E} \mathscr{W}^{-1}=\mathscr{C}_{\Phi(E)}
$$

ProOF: Let $E \in \Sigma$ with $\mu(E)>0$. Since $\mathscr{W} \mathscr{C}_{E} \mathscr{W}-1$ is a hermitian projection, there exists by Theorem (3.25) a $\mathscr{B}(K)$ valued function $P_{E}(\cdot)$ on $\Omega$ such that $P_{E}(\omega)$ is projection for almost all $\omega$ and $\mathscr{W} \mathscr{C}_{E} \mathscr{W}-1=\mathscr{M}_{P_{E}(.)}$.

Since $\mathscr{C}_{E}$ commutes with every operator of the form $\mathscr{M}_{H(\cdot)}$, where $H(\cdot)$ satisfies the conditions of Theorem (3.25), and since

$$
\mathscr{W} \mathscr{H}_{T} \mathscr{W}^{-1}=\mathscr{W} \mathscr{H}_{T_{1}} \mathscr{W}^{-1}+i \mathscr{W} \mathscr{M}_{T_{2}} \mathscr{W}^{-1},
$$

it follows from (3.25) that

$$
\mathscr{M}_{P_{E}(\cdot)} \mathscr{M}_{T}=\mathscr{M}_{T} \mathscr{M}_{\left.P_{\left.L^{(}\right)}\right)} \text {for each } T \in \mathscr{B}(K) \text {. }
$$

Thus for each $T \in \mathscr{B}(K)$ and $F \in L^{p}(\Omega, K)$ there exists a set $E(F, T) \in \Sigma$ with $\mu(E(F, T))=0$ such that

$$
P_{E}(\omega) T[F(\omega)]=T\left[P_{E}(\omega) F(\omega)\right] \text { for } \omega \in \Omega \backslash E(F, T) .
$$

By considering constant functions and using the separability of $K$ we can conclude from (4.4) that for each $T \in \mathscr{B}(K)$ there exists a set $E(T) \in \Sigma$ with $\mu(E(T))=0$ such that

$$
P_{E}(\omega) T(z)=T P_{E}(\omega)(z) \text { for each } z \in K \text { and } \omega \in \Omega \backslash E(T)
$$


Finally, since $K$ is separable, every rank 1 operator in $\mathscr{B}(K)$ can be approximated by a sequence of rank 1 operators from a given countable class. Hence there exists $E_{1} \in \Sigma$ with $\mu\left(E_{1}\right)=0$ such that for every rank 1 operator

$$
P_{E}(\omega) T(z)=T P_{E}(\omega)(z) \text { for each } z \in K \text { and } \omega \in \Omega \backslash E_{1} \text {. }
$$

The important thing to note about (4.6) is that $E_{1}$ does not depend on $z$ or $T$.

Now let us define $\Phi(E)=\left\{\omega \mid P_{E}(\omega) \neq 0\right\}$. Suppose there exists $E^{\prime} \subset \Phi(E) \cap\left(\Omega \backslash E_{1}\right)$ with $\mu\left(E^{\prime}\right)>0$ such that $P_{E}(\omega) \neq I$ for each $\omega \in E^{\prime}$. If $\omega_{1} \in E^{\prime}$ then there exists $z_{1}$ and $z_{2} \in K$, each with norm 1 , such $P_{E}\left(\omega_{1}\right) z_{1}=z_{1}$ and $P_{E}\left(\omega_{1}\right) z_{2}=0$. But if we define $T$ by $T z=\left(z, z_{1}\right) z_{2}$ we find that $P_{E}\left(\omega_{1}\right) T z_{1}=0$ while $T P_{E}\left(\omega_{1}\right) z_{1}=z_{2}$ ! This contradicts (4.6) and consequently $P_{E}(\omega)$ must be the identity operator on $K$ for $\omega \in \Phi(E)$. This completes the proof.

Lamperti (1958) calls a map $\Phi$ of $\Sigma$ onto $\Sigma$ (defined modulo sets of measure zero) a regular set isomorphism if (i) $\Phi(\widetilde{E})=\widetilde{\Phi(E)}$, (ii) $\Phi\left(U E_{i}\right)=U \Phi\left(E_{i}\right)$ and (iii) $\mu(\Phi(E))=0$ if and only if $\mu(E)=0$.

The following lemma is easy to verify.

(4.7) LeMma. The map $E \rightarrow \Phi(E)$ is a regular set isomorphism.

We will now show how the preceding results enable us to obtain a representation of $\mathscr{W}$. To that end let $z \in K$ with $|z|_{2}=1$ and $E$ a measurable set. If we define $z_{E}=\mathscr{C}_{E} z$, then

$$
\mathscr{W} z_{E}=\mathscr{C}_{\Phi(E)} \mathscr{W}(z)
$$

Since $\mathscr{W}$ is an isometry of $L^{p}(\Omega, K)$ we have

$$
\mu(E)=\left\|z_{\mathbb{E}}\right\|_{p}^{p}=\int_{\Phi(E)}|\mathscr{W}(z)|_{2}^{p} d \mu .
$$

Moreover, since the measure $\nu(E)=\mu\left(\Phi^{-1}(E)\right)$ is absolutely continuous with respect to $\mu$ we have

$$
\mu(E)=\nu(\Phi(E))=\int_{\Phi(E)}\left(\frac{d \nu}{d \mu}\right) d \mu .
$$

Comparing (4.9) and (4.11) we have by the uniqueness of the Radon-Nikodym derivative that there exists $E_{z} \subset \Omega$ with $\mu\left(E_{z}\right)=0$ such that

$$
|\mathscr{W}(z)|_{2}^{p}=\left(\frac{d \nu}{d \mu}\right) \text { for } \omega \in \Omega \backslash E_{z} .
$$

By an argument analogous to that preceding Theorem (3.25) it can be shown that there exists a $\mathscr{B}(K)$ valued function $U(\cdot)$ such that $U(\omega)$ is a surjective isometry for almost all $\omega$ and

$$
\mathscr{W}(z)(\omega)=\left(\frac{d \nu}{d \mu}\right)^{1 / p}(\omega) U(\omega) z
$$


Now using the fact that the vector simple functions are dense in $L^{p}(\Omega, K)$ we have:

(4.14) THEOREM (Cambern). Let $\mathscr{W}$ be a surjective isometry of $L^{p}(\Omega, K), 1 \leqslant p<\infty$, $p \neq 2$. Then there exists a regular set isomorphism $\Phi$ of the $\sigma$-algebra $\Sigma$ of measurable sets onto itself (defined modulo null sets), a scalar valued function $h$ defined on $\Omega$ satisfying $|h|^{p}=d v / d \mu$, and a $\mathscr{B}(K)$ valued function $U$ defined on $\Omega$ such that $U(\omega) z$ is measurable for each $z \in K$ and $U(\omega)$ is a surjective isometry of $K$ for almost all $\omega$ such that for $F \in L^{p}(\Omega, K)$,

$$
(\mathscr{W} F)(\omega)=U(\omega) h(\omega) \Phi(F)(\omega) .
$$

Conversely, every map $\mathscr{W}$ of this form is a surjective isometry of $L^{p}(\Omega, K)$.

REMARKs. Theorem (4.14) generalizes the theorem of Lamperti (1958). The proof given by Cambern does not rely on the theory of hermitian operators but does require the use of Lamperti's theorem.

Cambern asked whether a similar characterization can be given for the surjective isometries of $L^{p}(\Omega, B)$ for the case that $B$ belongs to a suitable class of finite dimensional Banach spaces. The following example shows that even when $B$ is two dimensional, the theorem does not hold.

EXAMPLE. Let $(\Omega, \Sigma, \mu)$ be a finite measure space and consider $L^{p}\left(\Omega, l_{(2)}^{p}\right)$. Let $\Phi_{1}, \Phi_{2}$ be distinct regular set isomorphisms with $h_{k}=\left(d \nu_{k} / d \mu\right)^{1 / p}$ where $v_{k}(E)=\mu\left(\Phi_{k}^{-1}(E)\right)$ for $E \in \Sigma$ and $k=1,2$. If $F \in L^{p}\left(\Omega, l_{(2)}^{p}\right)$ then $F(\cdot)=\left(F_{1}(\cdot), F_{2}(\cdot)\right)$. Let $(\mathrm{TF})(\omega)=\left(h_{1}(\omega) \Phi_{1}(F)(\omega), h_{2}(\omega) \Phi_{2}(F)(\omega)\right)$. It follows from Lamperti's theorem (1958) that $T$ is an onto isometry of $L^{p}\left(\Omega, l_{(2)}^{p}\right)$. Clearly $T$ is not of the form (4.15).

The techniques used to prove (4.14) can be extended to the case $L^{p}(\Omega, B)$ when $B$ is a separable smooth Banach space having the following property: If $P$ is a projection such that $P T=T P$ for every hermitian $T$ then $P$ is the identity on $B$. We note, however, that this condition seems to be very restrictive. It does not hold even when $B=l^{p}(2)$. This possibly accounts for the existence of an isometry such as the one given in the example above. It is tempting to conjecture that this restrictive condition holds only when $B$ is a Hilbert space. In any case it would be interesting to characterize the class of Banach spaces for which the condition holds.

\section{Adjoint abelian operators on $L^{p}(\Omega, K)$}

Stampfli (1969) introduced the notion of an adjoint abelian operator on a Banach space. An operator $\mathscr{S}$ on a complex Banach space $X$ is said to be adjoint abelian if there is a semi-inner product [,] compatible with the norm on $X$ such that

$$
[\mathscr{S} F, G]=[F, \mathscr{S} G] \text { for all } F, G \in X
$$


If $X$ is a Hilbert space, the adjoint abelian operators coincide with the usual hermitian operators.

Stampfli (1969) left as an open question as to whether every adjoint abelian operator on a weakly complete Banach space is scalar. In Fleming and Jamison (1976) we showed that this is indeed the case for the classical spaces $L^{p}$ and $\mathscr{C}(K)$, $K$ compact metric. We now show that this is the case for $L^{p}(\Omega, K)$.

If $\mathscr{S}$ is an adjoint abelian operator on $L^{p}(\Omega, K)$ then $\mathscr{S}^{2}$ is hermitian as well as adjoint abelian and hence there is a $\mathscr{B}(K)$ valued function $H(\cdot)$ satisfying the conditions of Theorem (3.25) such that $\mathscr{S}^{2}=\mathscr{M}_{H(.)}$. Since $\mathscr{M}_{H(.)}$ is adjoint abelian it follows (5.1) and (3.1) that for each $G \in L^{p}(\Omega, K)$ there exists a set $E_{a} \in \Omega$ with $\mu\left(E_{G}\right)=0$ and

$$
\frac{|H(\omega) G(\omega)|_{2}}{|G(\omega)|_{2}}=\frac{\left\|\mathscr{M}_{H(\cdot)} G\right\|_{p}}{\|G\|_{p}} \text { for } \omega \in \operatorname{supp}(G) \backslash E_{G} \cup E_{H},
$$

where

$$
E_{H}=\{\omega \mid H(\omega) \neq 0\} .
$$

By considering constant functions and using the separability of $K$ we can conclude from (5.2) that there exists $E_{0} \subset \Omega$ with $\mu\left(E_{0}\right)=0$ such that

$$
|H(\omega) z|_{2}=\left\|\mathscr{M}_{H(\cdot)} z\right\|_{p} \text { for } \omega \in \Omega \backslash\left(E_{0} \cup E_{H}\right) \text {. }
$$

It is now an easy matter to show that there is a fixed constant $\lambda$ such that

$$
\begin{array}{r}
|H(\omega) z|_{2}=\lambda \text { for every } z \in K \text { with }|z|_{2}=1 \\
\text { and for all } \omega \in \Omega \backslash\left(E_{0} \cup E_{H}\right) .
\end{array}
$$

Hence $\lambda^{-1} H(\omega)$ is an isometry for $\omega \in \Omega \backslash\left(E_{0} \cup E_{H}\right)$. Furthermore, it follows from (5.2) and (5.4) that $\mu\left(E_{H}\right)=0$. Therefore, if an operator is adjoint abelian on $L^{p}(\Omega, K)$, its square must be a multiple of an isometry. There is more that can be said.

(5.5) THEOREM. A linear operator $\mathscr{S}$ on $L^{p}(\Omega, K)$ is adjoint abelian on $L^{p}(\Omega, K)$, $1<p<\infty, p \neq 2$ if and only if there is a real constant $\lambda$ and an isometry $\mathscr{W}$ such that $\mathscr{W}^{2}=I$ and

$$
\mathscr{S}=\lambda \mathscr{W} .
$$

Proof. The sufficiency follows from Theorem 6 of Fleming and Jamison (1976). To prove the necessity, let $\mathscr{S}$ be an adjoint abelian operator. We may suppose that $\|\mathscr{S}\|=1$. Then by the arguments given above there exists an isometry $\mathscr{W}_{1}$ such that $\mathscr{S}^{2}=\mathscr{W}_{1}$ and by Theorem 1 of Stampfli (1969) it follows that $\|\mathscr{S}\|^{2}=\left\|\mathscr{S}^{2}\right\|=1$. Thus $\|\mathscr{S} F\|_{p} \leqslant 1$ for any $F$ in $L^{p}(\Omega, K)$ of norm 1 . If we suppose that there exists $F$ in $L^{p}(\Omega, K)$ of norm 1 such that $\|\mathscr{S} F\|_{p}<1$ then we see that

$$
1=\left\|\mathscr{S}^{2} F\right\|_{p} \leqslant\|\mathscr{S}\|\|\mathscr{S} F\|_{p} \leqslant\|\mathscr{S} F\|_{p}<1
$$


which is absurd. Therefore $\mathscr{S}$ is an isometry. From Theorem 6 of Fleming and Jamison (1976) there exists an isometry $\mathscr{W}$ of $L^{p}(\Omega, K)$ such that $\mathscr{W}^{2}=I$ and $\mathscr{S}=\mathscr{W}$. This completes the proof.

The next result follows from Corollary 4(a) of Stampfli (1969).

(5.8) COROLLARY. Every adjoint abelian operator on $L^{p}(\Omega, K)$ is a scalar operator.

REMARK: It is possible to give a more complete description of adjoint abelian operators on $L^{p}(\Omega, K)$ using Theorem (4.14) and arguments similar to those used in Fleming and Jamison (1976).

\section{References}

E. Berkson and A. Sourour (1974), "The hermitian operators on some Banach spaces", Studia Math. 52, 33-41.

M. Cambern (1974), “The isometries of $L^{p}(X, K)$ ", Pacific J. Math. 55, 9-17.

N. Dunford and J. T. Schwartz (1958), Linear Operators, Part 1 (Interscience, New York).

R. J. Fleming and J. E. Jamison (1974a), "Hermitian and adjoint abelian operators on certain Banach spaces", Pacific J. Math. 52 (1), 67-84.

R. J. Fleming and J. E. Jamison (1974b), "Isometries of certain Banach spaces", J. London Math. Soc. (2), 9, 121-127.

R. J. Fleming and J. E. Jamison (1976), "Adjoint abelian operators on $L^{p}$ and $C(K)$ ", Trans. Amer. Math. 217, 87-98.

E. Hille and R. Phillips (1957), Functional Analysis and Semi-groups (Colloquium Publications, Vol. 31, Providence, R.I.).

D. Koehler and P. Rosenthal (1970), "On isometries of normed linear spaces", Studia Math. 38, 215-218.

J. Lamperti (1958), “On the isometries of certain function spaces", Pacific J. Math. 2, 459-466.

S. Lang (1969), Analysis II (Addison-Wesley, Reading, Mass.).

G. Lumer (1961), "Semi-inner-Product Spaces", Trans. Amer. Math. Soc. 100, 26-43.

G. Lumer (1963), "Isometries of reflexive Orlicz spaces", Ann. Inst. Fourier, Grenoble, 13, 99-109.

J. G. Stampfli (1969), “Adjoint Abelian operators on Banach spaces”, Canadian J. Math. 31, 505-512.

K. W. Tam (1969), "Isometries of certain function spaces", Pacific J. Math. 31, 233-246.

Department of Mathematics

Memphis State University

Memphis Tennessee 38152

USA 